\title{
Impact of vacancies and impurities on ferroelectricity in PVD- and ALD-grown $\mathrm{HfO}_{2}$ films
}

Cite as: Appl. Phys. Lett. 118, 032903 (2021); https://doi.org/10.1063/5.0035686

Submitted: 30 October 2020 . Accepted: 02 January 2021 . Published Online: 19 January 2021

(D) Lutz Baumgarten, (D) Thomas Szyjka, (D) Terence Mittmann, et al.

\section{COLLECTIONS}

Paper published as part of the special topic on Ferroelectricity in Hafnium Oxide: Materials and Devices
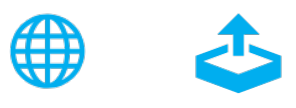

\section{ARTICLES YOU MAY BE INTERESTED IN}

Polarization switching in thin doped $\mathrm{HfO}_{2}$ ferroelectric layers

Applied Physics Letters 117, 262904 (2020); https://doi.org/10.1063/5.0035100

Ferroelectricity in hafnium oxide thin films

Applied Physics Letters 99, 102903 (2011); https://doi.org/10.1063/1.3634052

Impact of area scaling on the ferroelectric properties of back-end of line compatible $\mathrm{Hf}_{0.5} \mathrm{ZrO} \mathrm{O}_{2}$ and $\mathrm{Si}: \mathrm{HfO}_{2}$-based MFM capacitors

Applied Physics Letters 118, 062904 (2021); https://doi.org/10.1063/5.0035650

\section{Challenge us.}

What are your needs for periodic signal detection?

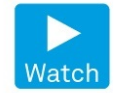

Zurich

Instruments

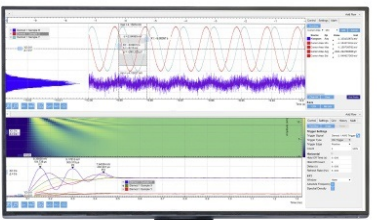

(0 


\title{
Impact of vacancies and impurities on ferroelectricity in PVD- and ALD-grown $\mathrm{HfO}_{2}$ films
}

\author{
Cite as: Appl. Phys. Lett. 118, 032903 (2021); doi: 10.1063/5.0035686 \\ Submitted: 30 October 2020 - Accepted: 2 January 2021 • \\ Published Online: 19 January 2021
}

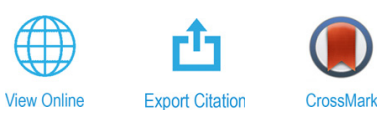

Lutz Baumgarten,, ${ }^{1, a)}$ (D) Thomas Szyjka,' (iD Terence Mittmann, ${ }^{2}$ (D Monica Materano, ${ }^{2}$ (D) Yury Matveyev, ${ }^{3}$ Christoph Schlueter, ${ }^{3}$ (D) Thomas Mikolajick, ${ }^{2,4}$ (iD) Uwe Schroeder, ${ }^{2}$ (D) and Martina Müller ${ }^{5}$

\author{
AFFILIATIONS \\ ${ }^{7}$ Forschungszentrum Jülich GmbH, Peter Grünberg Institut (PGI-6), 52425 Jülich, Germany \\ ${ }^{2} \mathrm{NaMLab}$ gGmbH, Noethnitzer Str.. 64a, 01187 Dresden, Germany \\ ${ }^{3}$ Deutsches Elektronen-Synchrotron, Notkestraße 85, 22607 Hamburg, Germany \\ ${ }^{4}$ Chair of Nanoelectronic, TU Dresden, 01062 Dresden, Germany \\ ${ }^{5}$ Fachbereich Physik, Universität Konstanz, 78457 Konstanz, Germany
}

Note: This paper is part of the Special Topic on Materials and Devices Utilizing Ferroelectricity in Halfnium Oxide.

a) Author to whom correspondence should be addressed: l.baumgarten@fz-juelich.de

\begin{abstract}
We investigate the emerging chemical states of $\mathrm{TiN} / \mathrm{HfO}_{2} / \mathrm{TiN}$ capacitors and focus especially on the identification of vacancies and impurities in the ferroelectric $\mathrm{HfO}_{2}$ layers, which are produced either by physical vapor deposition (PVD) or atomic layer deposition (ALD). Depending on the specific growth conditions, we identify different mechanisms of oxygen vacancy formation. Corresponding spectral features are consistently observed for all $\mathrm{HfO}_{2}$ - and TiN-related core levels by hard x-ray photoelectron spectroscopy (HAXPES). In ALDgrown samples, we find spectral signatures for the electronic interaction between oxygen vacancies and nitrogen impurities. By linking the HAXPES results to electric field cycling experiments on the $\mathrm{TiN} / \mathrm{HfO}_{2} / \mathrm{TiN}$ capacitors, we discuss possible formation mechanisms and stabilization of the ferroelectric $\mathrm{HfO}_{2}$ phase directly related to specific PVD or ALD conditions.
\end{abstract}

Published under license by AIP Publishing. https://doi.org/10.1063/5.0035686

$\mathrm{HfO}_{2}$ is a key multifunctional material for current device technology. Recently, it is also been successfully used as a ferroelectric or memristive material in nonvolatile memory devices and for novel neuromorphic computing concepts. ${ }^{1-3}$ Atomic layer deposition (ALD) has become the most widely employed growth process since it allows for a precise thickness control on the level of single atomic layers at CMOS-compatible low deposition temperatures.

However, ALD-grown $\mathrm{HfO}_{2}$ thin films can contain a significant amount of impurities like carbon or nitrogen if the decomposition of the precursor gas in ozone was incomplete or removed ligands readsorb on the surface. The impact of $\mathrm{N}$ or $\mathrm{C}$ impurities on the $\mathrm{HfO}_{2}$ thin film ferroelectric properties is not fully clarified to date.

As an alternative technique, physical vapor deposition (PVD) by sputtering from an $\mathrm{HfO}_{2}$ target was explored to gain even better control on the stoichiometry and defect concentration in $\mathrm{HfO}_{2}$ thin films.

Oxygen vacancies are recognized as those defects, which have a positive impact on the stabilization of the ferroelectric orthorhombic phase in undoped $\mathrm{HfO}_{2} \cdot{ }^{4-6}$ Here, we present a comparative study of both ALD- and PVD-grown $\mathrm{HfO}_{2}$ thin films interfaced with TiN bottom/top electrodes (BE/TEs) integrated into TiN/HfO $2 /$ TiN capacitors. We apply hard $\mathrm{x}$-ray photoelectron spectroscopy (HAXPES) as an equally depth-sensitive and element-specific chemical characterization technique, ${ }^{7,8}$ which allows us to gain a comprehensive picture of the chemical state of the $\mathrm{HfO}_{2}$ layers. In our analysis, we focus, in particular, on identifying spectral signatures of oxygen vacancies and nitrogen/carbon impurities. We correlate these spectroscopic findings with electrical measurements of the remanent polarization of the ALD- and PVD-grown $\mathrm{TiN} / \mathrm{HfO}_{2} / \mathrm{TiN}$ capacitors. In this way, possible mechanisms of stabilization of the ferroelectric $\mathrm{HfO}_{2}$ phase, resulting from the specific PVD or ALD conditions, are identified.

Sample preparation for PVD and ALD samples is sketched in Fig. 1. For further details, see the supplementary material.

In order to access chemical information from the TiN/ $/ \mathrm{HfO}_{2}$ bottom interface, we performed HAXPES measurements on interstitial areas between the etched TiN top electrodes. The observation of interfacial $\mathrm{TiO}_{2}$ in the case of PVD (see Fig. 2) arises from the oxygen supply $\dot{m}$ provided before the in situ PVD process (see Fig. 1, left). ${ }^{9}$ The effective thickness of the $\mathrm{TiO}_{2}$ intralayer saturates in a self-limiting 


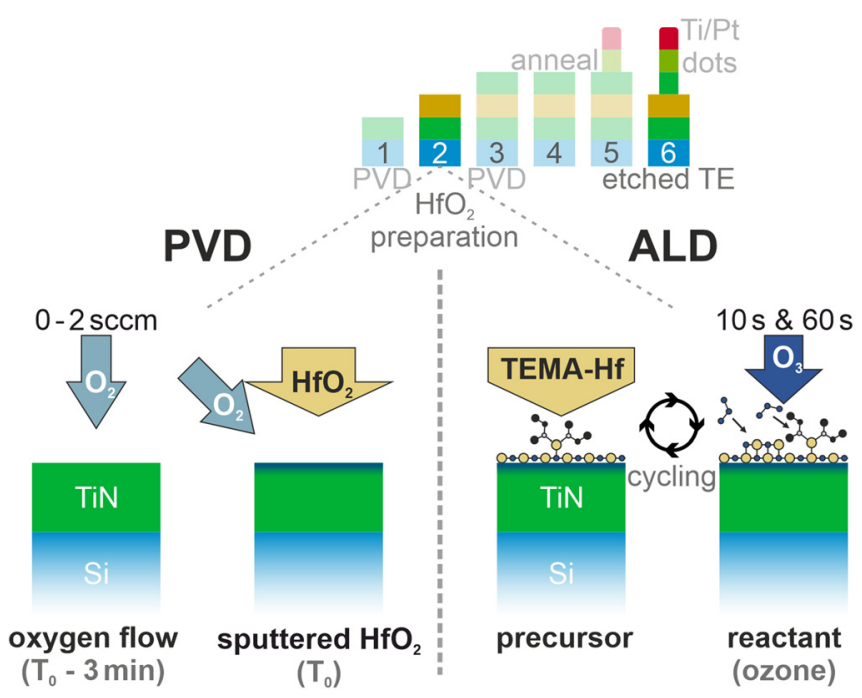

FIG. 1. Process schematics for PVD and ALD of ferroelectric $\mathrm{HfO}_{2}$ in $\mathrm{TiN} / \mathrm{HfO} / 2 / \mathrm{TiN}$ capacitors: (Left) Using PVD, $\mathrm{HfO}_{2}$ is sputtered in an additional oxygen flow of $\dot{m}=0$ or $2 \mathrm{sccm}$, respectively. Before the $\mathrm{HfO}_{2}$ evaporation, the $\mathrm{O}_{2}$ flux is stabilized for $t=3 \mathrm{~min}$. (Right) Using $\mathrm{ALD}$, layer-by-layer growth of $\mathrm{HfO}_{2}$ is accomplished by an alternating supply (cycling) of TEMA-Hf as the precursor and reactive ozone as the reactant, applying pulse times of $t_{\mathrm{O}_{3}}=10$ and $60 \mathrm{~s}$, respectively.

process at about $3.5( \pm 0.5) \mathrm{nm}$. As a consequence, the chemical exchange between the TiN electrode and the $\mathrm{HfO}_{2}$ is strongly reduced or even suppressed by the $\mathrm{TiO}_{2}$ intralayer, which serves as a chemical buffer. $^{9}$ For the ALD-grown samples, we also observed spectral contributions of interfacial $\mathrm{TiO}_{2}$ (see Fig. 2). Here, a sizeable surface oxidation is a result of an exposure to air for at least $24 \mathrm{~h}$ before ALD growth of $\mathrm{HfO}_{2}$. Indeed, the $\mathrm{TiO}_{2}$-related spectral weights of both $\mathrm{ALD}_{10 \mathrm{~s}}$ and $\mathrm{ALD}_{60 \mathrm{~s}}$ samples are comparable to those of the $\mathrm{PVD}_{2 \mathrm{sccm}}$ sample at $\dot{m}=2 \mathrm{sccm}$. Independent of its chemical origin, however, the $\mathrm{TiO}_{2}$ layer serves as a chemical buffer also in ALD-grown samples.

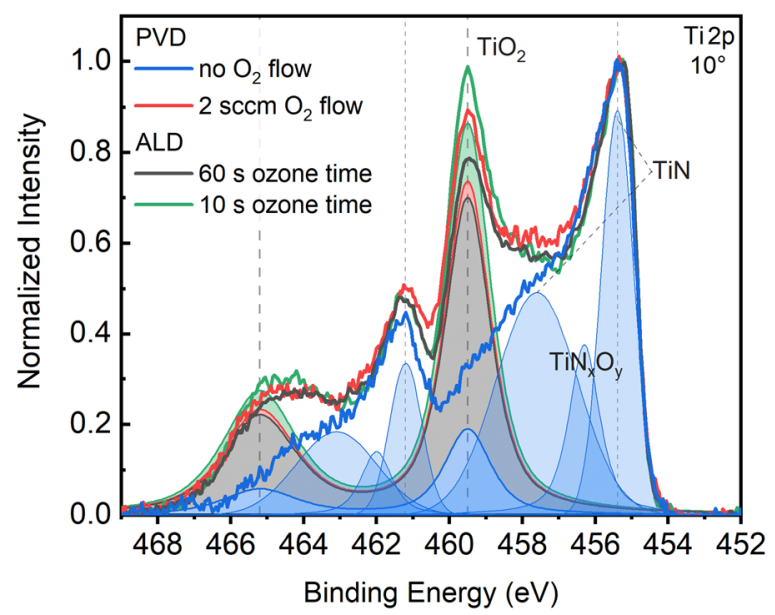

FIG. 2. HAXPES spectra of the Ti $2 p$ core levels for PVD- and ALD-grown samples. An extended interfacial $\mathrm{TiO}_{2}$ formation is observed for both $A L D_{10 \mathrm{~s}}$ and $A L D_{60 \text { s }}$ samples as well as for $P V D_{2 s c c m}$, but not for the $P V D_{0 s c c m}$ sample. $T_{10}$ serves as a chemical buffer between the $\mathrm{HfO}_{2}$ and TiN bottom electrode.
Figure 3 shows the Hf $4 \mathrm{f}$ core levels for both sets of PVD- and ALD-grown samples. The intensity is normalized to the $\mathrm{Hf}_{4} \mathrm{f}_{5 / 2}$ peak maximum. We find the $\mathrm{PVD}_{0 \text { sccm }}$ and $\mathrm{PVD}_{2 \text { sccm }} \mathrm{Hf} 4 \mathrm{f}$ peak maxima located at higher binding energies compared to the spectra of both ALD-grown samples. The largest binding energy shift $\Delta \mathrm{E}_{\mathrm{B}}=270$ $\mathrm{meV}$ is observed between the $\mathrm{PVD}_{0 \mathrm{sccm}}$ and $\mathrm{ALD}_{10 \mathrm{~s}}$ samples. It should be noted that we observe these binding energy shifts between PVD- and ALD-grown samples in all spectral features, which are related to the $\mathrm{HfO}_{2}$ layer (also see the supplementary material). On the other hand, core level features of the TiN electrodes or the $\mathrm{TiO}_{2-x}$ interface layer do not show any relative binding energy shift $\Delta \mathrm{E}_{\mathrm{B}}$.

Next, we determined the $\mathrm{Hf} 4 \mathrm{f}$ difference spectra relative to the $\mathrm{ALD}_{60 \text { s }}$ sample (see the supplementary material). They reveal additional Hf $4 \mathrm{f}$ contributions at lower binding energies originating from $\mathrm{Hf}^{3+}$, which are attributed to the presence of oxygen vacancies $V_{0}{ }^{9-13}$ For a quantitative analysis, the Hf $4 \mathrm{f}$ spectra were fitted consistently by two doublets. The fits for the $\mathrm{Hf}^{3+}$ contributions are shown as shaded peaks in the lower part of Fig. 3 , and the particular $\mathrm{Hf}^{3+} / \mathrm{Hf}^{4+}$ areal intensity ratios are given in Table I.

As expected, we find a sizeable $\mathrm{Hf}^{3+}$ component for the $\mathrm{PVD}_{0 \mathrm{sccm}}$ sample and only a small one for $\mathrm{PVD}_{2 \mathrm{sccm}}$, while both ALD samples are nearly free of $\mathrm{Hf}^{3+}$. For $\mathrm{ALD}_{10}$ s, we still find a tiny $\mathrm{Hf}^{3+}$ component that disappears completely after a longer ozone dose in $\mathrm{ALD}_{60}$, in agreement with recent findings. ${ }^{14}$

Figure 4 shows the Hf $4 \mathrm{~s}$ and $\mathrm{O}$ 1s core levels, which have been normalized to the $\mathrm{Hf} 4 \mathrm{~s}$ peak. The $\mathrm{O} 1 \mathrm{~s}$ peak intensity shows a gradual increase from $\mathrm{PVD}_{0 \mathrm{sccm}}, \mathrm{PVD}_{2 \mathrm{sccm}}, \mathrm{ALD}_{60 \mathrm{~s}}$ to $\mathrm{ALD}_{10 \mathrm{~s}}$. The O 1s/Hf $4 \mathrm{~s}$ intensity ratios obtained from an integration over the normalized spectra are given in Table I. For PVD samples, a larger additional oxygen supply leads to a larger oxygen intensity. Moreover, a larger ozone dose $\left(\mathrm{ALD}_{60 \mathrm{~s}}\right)$ results in a lower oxygen spectral intensity compared to the $\mathrm{ALD}_{10 \mathrm{~s}}$ sample.

The O/Hf intensity ratio may decrease for two reasons: First, undesired processes such as the readsorption of organic precursor ligands can likely occur for longer ozone times and may modify the $\mathrm{O} / \mathrm{Hf}$ ratio in a poorly controllable manner. Second, the relative O/Hf

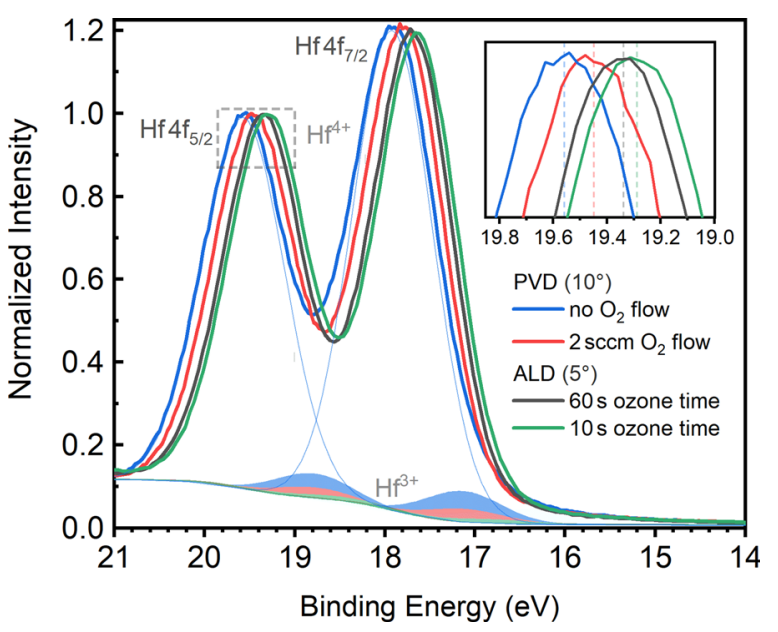

FIG. 3. HAXPES spectra of the $\mathrm{Hf} 4 \mathrm{f}$ core levels for PVD- and ALD-grown samples. The $P V D_{0,2 \mathrm{sccm}}$ samples are energetically shifted relative to the $A L D_{10,60 \mathrm{~s}}$ samples. Shaded areas correspond to $\mathrm{Hf}^{3+}$ components present in the $\mathrm{PVD}_{0,2 \mathrm{sccm}}$ samples. 
TABLE I. Area intensity ratios $\mathrm{Hf}^{3+} / \mathrm{Hf}^{4+}$ of the $\mathrm{Hf} 4 \mathrm{f}$ spectra (Fig. 3). Area intensity ratios of $01 \mathrm{~s}, \mathrm{C} 1 \mathrm{~s}$, and the neighboring $\mathrm{Hf}$ core levels (Figs. 4 and 5). All values are in $\%$.

\begin{tabular}{lccc}
\hline \hline & Hf 4f: $\mathrm{Hf}^{3+} / \mathrm{Hf}^{4+}$ & $\mathrm{O} 1 \mathrm{~s} / \mathrm{Hf} 4 \mathrm{~s}$ & $\mathrm{C} 1 \mathrm{~s} / \mathrm{Hf} 4 \mathrm{~d}$ \\
\hline $\mathrm{PVD}_{0 \text { sccm }}$ & $6.2( \pm 0.2)$ & 1.64 & 0.18 \\
$\mathrm{PVD}_{2 \mathrm{sccm}}$ & $2.7( \pm 0.1)$ & 1.73 & 0.18 \\
$\mathrm{ALD}_{60 \mathrm{~s}}$ & $0.0( \pm 0.1)$ & 1.81 & 0.50 \\
$\mathrm{ALD}_{10 \mathrm{~s}}$ & $0.8( \pm 0.1)$ & 1.89 & 1.24 \\
\hline \hline
\end{tabular}

ratio may also decrease by increasing the absolute $\mathrm{Hf}$ content, which is most likely the case for the $\mathrm{ALD}_{60}$ s sample. We note that such large $\mathrm{O}_{3}$ doses (60 s) are typically not applied in ALD, and we use it here for the purpose of studying the impact of impurities.

Furthermore, the $\mathrm{O}$ 1s spectra show a slight increase in intensity at a binding energy of about $533 \mathrm{eV}$ (valley region) for both ALD spectra as compared to PVD. This intensity increase indicates a satellite peak, which is split off from the main $\mathrm{O} 1 \mathrm{~s}$ peak by about $1.8 \mathrm{eV}$ (see the supplementary material, Sec. II B). This feature has been discussed in the literature as a signature for an oxygen vacancy $V_{0}$ in different oxide materials, ${ }^{15-20}$ with either a $1+$ or $2+$ valency. ${ }^{12,13,21}$

A major difference between ALD- and PVD-grown $\mathrm{HfO}_{2}$ thin films results from using a metal-organic precursor molecule (TEMA$\mathrm{Hf}$ ) in the ALD case. We find clear signatures of $\mathrm{C}$ and $\mathrm{N}$ impurities incorporated in the ALD-grown $\mathrm{HfO}_{2}$ films, as discussed in the following.

In Fig. 5, the Carbon C 1s core levels recorded at different photoelectron emission angles are shown for (a) ALD- and (b) PVD-grown samples. All spectra were normalized to the neighboring $\mathrm{Hf} 4 \mathrm{~d}$ peaks (not shown). The amount of carbon detected in the PVD-grown $\mathrm{HfO}_{2}$ layers is, as expected, very low. Moreover, the $\mathrm{C} 1 \mathrm{~s}$ peaks do not show any dependence either on the oxygen flow during PVD growth or on the emission angle of the measurement. This finding indicates a homogeneous distribution of carbon impurities within the sample stack, which most likely originates from residuals in the vacuum chamber present throughout the deposition.

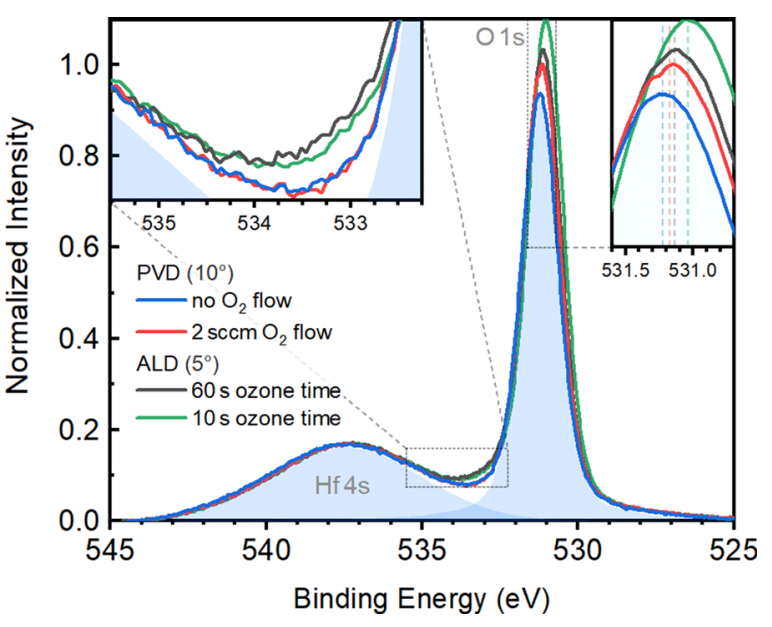

FIG. 4. Normalized HAXPES spectra of the $\mathrm{Hf} 4 \mathrm{~s}$ and $\mathrm{O}$ 1s core levels for PVDand ALD-grown samples. (Inset, upper left) The valley region between the $\mathrm{Hf} 4 \mathrm{~s}$ and $\mathrm{O} 1 \mathrm{~s}$ core level reveals larger intensity for the ALD samples. (Inset, upper right) The peak maximum of the 0 1s core level varies for either PVD or ALD-grown samples, respectively.

Clear variations are, however, observable in the $\mathrm{C}$ 1s core level of the ALD-grown samples: The C 1 s peak intensity is reduced to $\sim 40 \%$ while increasing the $\mathrm{O}_{3}$ dosage from 10 to $60 \mathrm{~s}$. Moreover, the $\mathrm{C} 1 \mathrm{~s}$ peak intensity shows a strong decrease at a larger surface sensitivity of $41^{\circ}$. Hence, we conclude on an accumulation of carbon impurities toward the bottom TiN electrode [see the inset of Fig. 5(a)]. This finding suggests that the cycled ALD reaction of the TEMA-Hf precursor molecule with $\mathrm{O}_{3}$ is less effective on a $\mathrm{TiO}_{2}$ surface, i.e., at the beginning of the $\mathrm{HfO}_{2}$ growth process, thereby enhancing the amount of organic $\mathrm{C}$ residuals in the first $\mathrm{HfO}_{2}$ layers.

The HAXPES spectra of the nitrogen $\mathrm{N}$ 1s core levels, shown in Fig. 6 for (a) ALD and (b) PVD samples, are both dominated by the $\mathrm{Ti}-\mathrm{N}$ contribution from the bottom electrode and $\mathrm{O}-\mathrm{Ti}-\mathrm{N}$ contribution from the $\mathrm{TiN} / \mathrm{HfO}_{2}$ interface. ${ }^{9,10}$ The peak shoulder at (a)

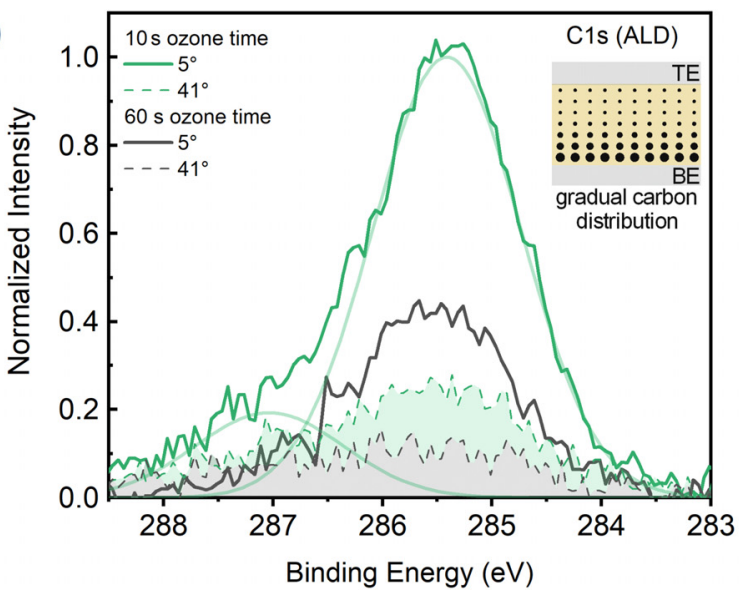

(b)

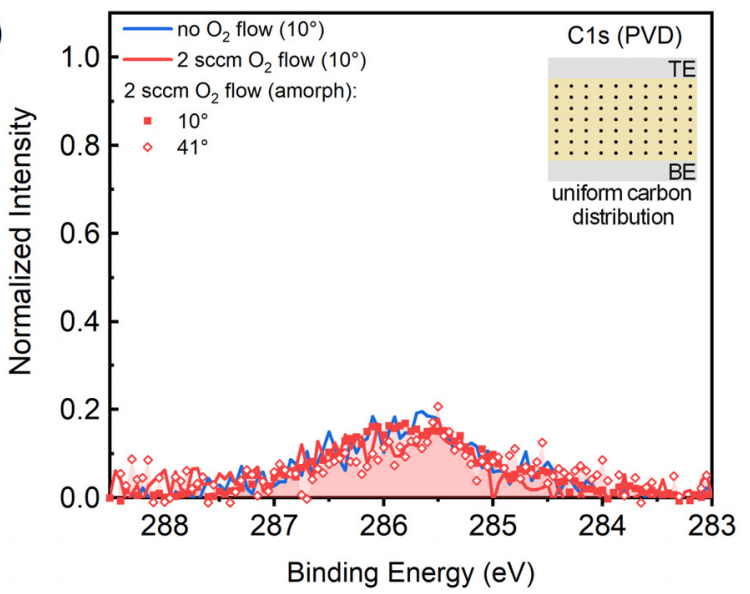

FIG. 5. HAXPES spectra of the C 1s core levels for (a) ALD- and (b) PVD-grown samples. The C content in ALD samples depends on ozone dosage and gradually increases toward the bottom TiN interface. PVD samples show only a very small, uniformly distributed, and growth-independent amount of $\mathrm{C}$ impurities. 
(a)

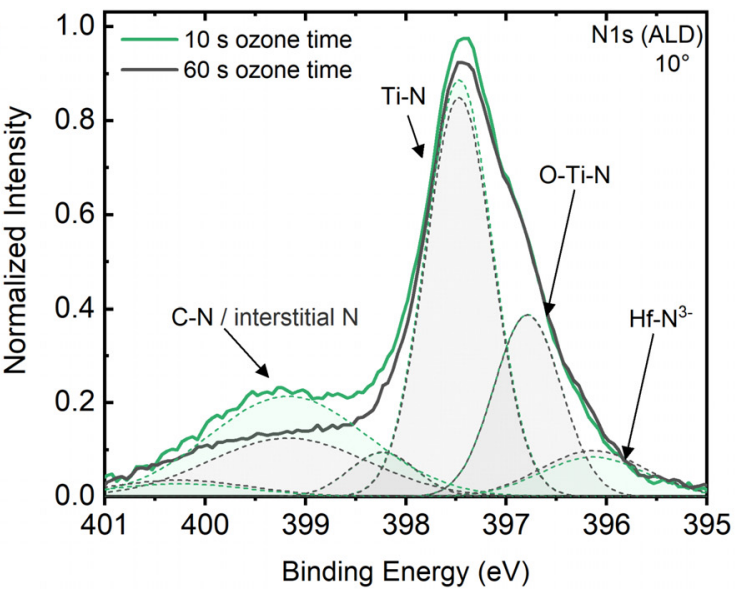

(b)

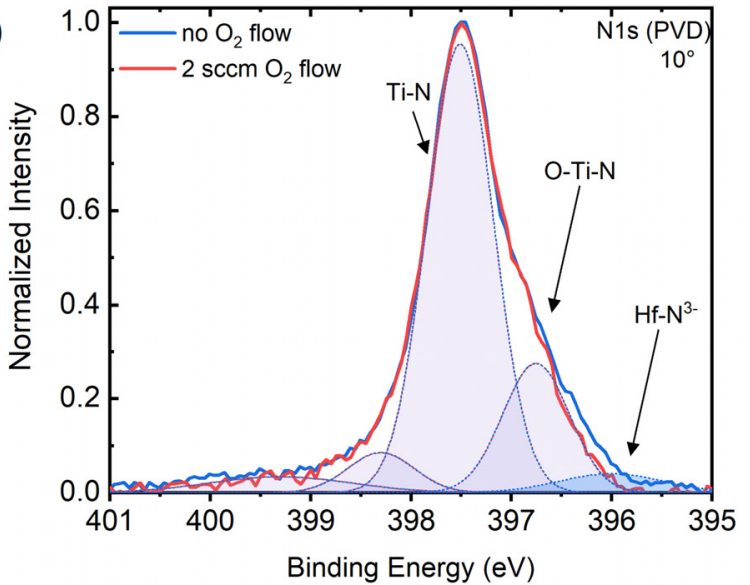

FIG. 6. HAXPES spectra of the N 1s core levels for (a) ALD- and (b) PVD-grown samples, dominated by Ti-N contribution from the bottom electrode and O-Ti-N contribution from the TiN/ $/ \mathrm{HfO}_{2}$ interface. Hf-N contributions are observed for all samples but $P V D_{2 s c m}$. (a) In ALD samples, C-N or interstitial N decreases with increasing ozone dose, as does the corresponding $C$ 1s spectra in Fig. 5(a).

$\mathrm{E}_{\mathrm{B}}=396 \mathrm{eV}$ is related to $\mathrm{Hf}^{4+}-\mathrm{N}$ coordination, ${ }^{22}$ whereas the broad emission around $\mathrm{E}_{\mathrm{B}}=399 \mathrm{eV}$ to $\mathrm{C}-\mathrm{N}$ bonds and interstitial Nitrogen contributions. $^{23,24}$

In the PVD samples shown in Fig. 6(b), the Hf-N component only appears for $\mathrm{PVD}_{0 \mathrm{sccm}}$ and is related to an $\mathrm{Hf}-\mathrm{N}$ formation at the $\mathrm{TiN} / \mathrm{HfO}_{2}$ interface, which is suppressed by the $\mathrm{TiO}_{2}$ intralayer formed in $\mathrm{PVD}_{2 \mathrm{sccm}}$ samples: Since an interface reaction in ALD samples is also protected by a $\mathrm{TiO}_{2}$ layer, the Hf-N component in ALD samples has to be associated with a residual impurity of the ALD process. The same applies to the $\mathrm{N}$ component around $\mathrm{E}_{\mathrm{B}}=399 \mathrm{eV}$ originating from $\mathrm{C}-\mathrm{N}$ or rather interstitial $\mathrm{N}$. This component shows hardly any angular dependence but is strongly reduced by increasing the $\mathrm{O}_{3}$ dose from $10 \mathrm{~s}$ to $60 \mathrm{~s}$. The intensity of the Hf-N component, however, remains basically constant for an increased $\mathrm{O}_{3}$ dose, which indicates a more stable bonding. From its angular dependence (see the supplementary material, Sect. IIC), we conclude on its accumulation at the bottom electrode.

Next, we discuss the experimental observations in terms of the interactions between oxygen vacancies $\left(\mathrm{V}_{0}\right)$ and impurities $(\mathrm{C}$ or $\mathrm{N})$.

Generally, the ferroelectric phase in $\mathrm{HfO}_{2}$ can be stabilized by defects. ${ }^{6}$ For PVD samples, the oxygen vacancies likely take this function. ${ }^{4}$ In HAXPES spectra, oxygen vacancies $V_{0}$ manifest themselves as a small $\mathrm{Hf}^{3+}$ component at the low binding energy side of $\mathrm{Hf}$ 4f spectra (see Fig. 3). The $\mathrm{Hf}^{3+}$ signature appears, if the two electrons left behind at the vacancy site from a removed neutral oxygen atom are distributed among two neighboring $\mathrm{Hf}^{4+}$ atoms.

The reduced $\mathrm{Hf}^{3+}$ component in the $\mathrm{PVD}_{2 \mathrm{sccm}}$ compared to $\mathrm{PVD}_{0 \mathrm{sccm}}$ originates from the larger amount of oxygen provided during growth, which fills up the vacancy sites. The emerging ferroelectric properties are fully consistent with this chemical state: Whereas the $\mathrm{PVD}_{0 \text { sccm }}$ sample is ferroelectric with $2 \mathrm{P}_{\mathrm{r}, 10^{5}}=18.2 \mathrm{uC} / \mathrm{cm}^{2}$, see also Fig. 7, the $\mathrm{PVD}_{2 \mathrm{sccm}}$ sample does not show a significant ferroelectric remanence, as discussed in our previous work. ${ }^{9}$

One might conclude that the absence of the $\mathrm{Hf}^{3+}$ component in both ALD Hf 4 f spectra (Fig. 3) should result in a reduced ferroelectric remanence. Yet, quite the contrary is the case: Both $\mathrm{ALD}_{10 \mathrm{~s}, 60 \mathrm{~s}}$ samples reveal a sizeable remanent polarization with $2 \mathrm{P}_{\mathrm{r}, 10^{5}}^{10 \mathrm{~s}}=13.8$ $\mu \mathrm{C} / \mathrm{cm}^{2}$ and $2 \mathrm{P}_{\mathrm{r}}^{60 \mathrm{~s}} 0^{5}=11.1 \mu \mathrm{C} / \mathrm{cm}^{2}$, respectively. This is remarkable because the $\mathrm{O} / \mathrm{Hf}$ ratio increases for the ALD samples compared to the PVD samples. Thus, we suggest that the role of the other impurities, i.e., carbon and nitrogen, needs to be taken into closer consideration.

Kim et al $^{24}$ argued that $\mathrm{C}$ impurities can also stabilize the ferroelectric $\mathrm{HfO}_{2}$ phase, and Rodenbücher et al. ${ }^{25}$ found that a high concentration of oxygen vacancies considerably lowers the formation energy of Hf-C bonds.

However, the C 1s spectra do not show signatures of such an Hf$\mathrm{C}$ contribution, and thus, we rule out a stabilization of the ferroelectric phase in $\mathrm{HfO}_{2}$ by such a process in the ALD samples.

Rather, we shall discuss the stabilization of the ferroelectric phase by the incorporation of nitrogen impurities into $\mathrm{HfO}_{2}$ thin films.

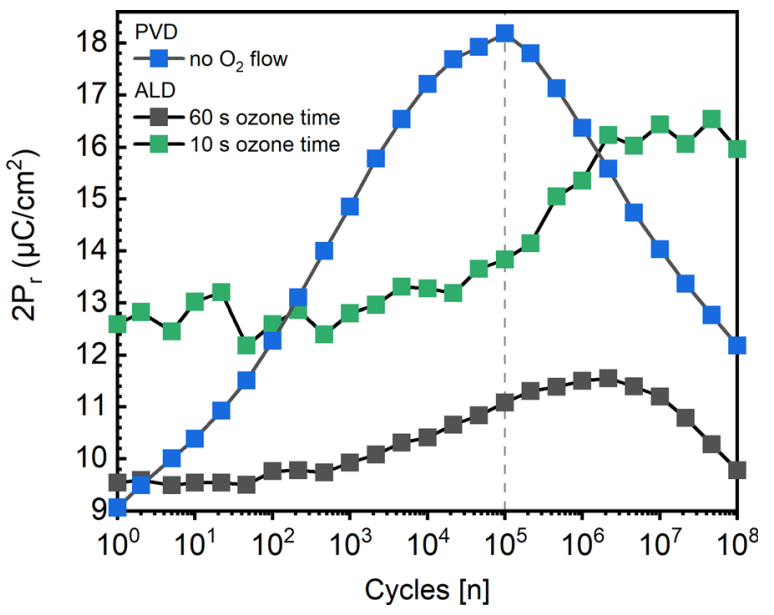

FIG. 7. Electric field cycling behavior of $\mathrm{TiN} / \mathrm{HfO}_{2} / \mathrm{TiN}$ capacitors, showing the double remanent polarization $2 \mathrm{P}_{\mathrm{r}}$ of $A L D_{10 \mathrm{~s}}, A L D_{60 \mathrm{~s}}$, and $\mathrm{PVD}_{0 \mathrm{sccm}}$ samples, which vanishes for $P V D_{2 s c c m}$ to $2 P_{r}=0$. 
Indeed, nitrogen has been reported both theoretically and experimentally to be the only anion dopant that can stabilize the ferroelectric $\mathrm{HfO}_{2}$ phase. $^{26,27}$ In their ab initio models, Umezawa et al. ${ }^{28,29}$ and Xiong et al. ${ }^{30}$ proposed an incorporation of nitrogen at the two oxygen nearest neighbor sites of a vacancy. This configuration was found to be energetically most favorable compared to other possible occupation sites for nitrogen, including the vacancy site itself, since two nitrogen will capture both electrons from the oxygen vacancy and form a closed shell $\mathrm{VN}_{2}$ complex. ${ }^{28,31,32}$ Locally, the valencies then amount to $\mathrm{V}_{O}^{2+}$ for the oxygen vacancy site, $\mathrm{N}^{3-}$ for nitrogen, and hafnium remains in a $\mathrm{Hf}^{4+}$ state.

On the experimental side, Lomenzo et al. concluded on the formation of this nitrogen-vacancy complex as an interface reaction at the $\mathrm{TaN} / \mathrm{HfO}_{2}$ interface. ${ }^{33}$ In our case, such an interface reaction can be excluded due to the passivation by the $\mathrm{TiO}_{2}$ interlayer.

This modeling is fully consistent with the HAXPES data of the ALD samples, which reveal vanishing of oxygen vacancy signatures $V_{0}$ in the Hf 4 f spectra and appearance of stable $\mathrm{Hf}^{4+}-\mathrm{N}$ bonds in the $\mathrm{N}$ $1 \mathrm{~s}$ spectra and vacancy signatures in the $\mathrm{O} 1 \mathrm{~s}$ spectra. Moreover, the incorporated $\mathrm{N}$ impurities may even compensate the reduced oxygen vacancy density by stabilizing the ferroelectricity in ALD samples. ${ }^{2,27}$

Next, we consider the peak shifts of $\mathrm{Hf} 4 \mathrm{f}$ and $\mathrm{O}$ 1s toward lower binding energies (Fig. 3). They can be attributed to the decreasing density of oxygen vacancy states within the bandgap. For the PVD samples, this is reflected by the decreasing $\mathrm{Hf}^{3+}$ component, caused by the replenishing of vacancy sites by oxygen atoms and the increasing $\mathrm{O}$ 1s intensity (Fig. 4). For the ALD samples, the larger shift of $\mathrm{Hf} 4 \mathrm{f}$ toward lower binding energies as compared to the PVD samples can also be attributed to a further decrease in neutral oxygen vacancy states within the bandgap. However, here the $\mathrm{N}$ impurities play an additional role in the electronic structure formation. The vacancies are not simply replenished but also electronically passivated by nitrogen impurities, thus forming a $\mathrm{VN}_{2}$ complex. The former $\mathrm{V}_{0}$ gap states are now shifted into the valence band. ${ }^{30}$

As highlighted before, oxygen vacancies are essential for stabilizing the ferroelectric phase in $\mathrm{HfO}_{2}$ films and also determine the onset of the wake-up effect during electric field cycling of the $\mathrm{TiN} / \mathrm{HfO}_{2} / \mathrm{TiN}$ capacitors. ${ }^{4,34,35}$ A key parameter is the mobility of the oxygen vacancies under the application of an electric field. It determines their spacial redistribution and increases the ferroelectric polarization during the wake-up cycling. Hence, a change in the mobility of oxygen vacancies should result in a sizable alteration of the electric wake-up behavior.

In the case of ALD-grown samples, the formation of a vacancyimpurity $\mathrm{VN}_{2}$ complex should result in a decreased mobility compared to vacancies $V_{0}$ in PVD-grown samples, due to the chemical bonding of the oxygen vacancy to the nitrogen impurity. ${ }^{30}$ Fully consistent with this assumption, indeed, the electric field cycling behavior of the PVD-grown samples compared to the ALD ones shows an essentially different shape (see Fig. 7). However, the PVD $_{0 \text { sccm }}$ sample reveals a pronounced increase in the remanent ferroelectric polarization $2 \mathrm{P}_{\mathrm{r}}$ during wake-up, which may be related to a high mobility of the oxygen vacancies within the $\mathrm{HfO}_{2}$ layer, like de-pinning of domains pinned to the defect states. Both ALD samples show only a minor increase and a smaller slope of the $2 \mathrm{P}_{\mathrm{r}}$ curves. Hence, this observation might be related to a decreased mobility of the $\mathrm{VN}_{2}$ complex formed in the ALD-grown $\mathrm{HfO}_{2}$ thin films compared to the oxygen vacancies $\mathrm{V}_{0}$ present in PVD-grown $\mathrm{HfO}_{2}$.
In summary, we presented an HAXPES study of TiN/ $/ \mathrm{HfO}_{2} / \mathrm{TiN}$ capacitors, for which the ferroelectric $\mathrm{HfO}_{2}$ layers were either prepared by PVD sputtering or by ALD deposition. A comparative picture of the emerging chemical states dependent on the specific PVD or ALD growth conditions was obtained, in particular of the $\mathrm{HfO}_{2}$ layers. The spectral signatures observed in the $\mathrm{Hf} 4 \mathrm{f}, \mathrm{O} 1 \mathrm{~s}$, and $\mathrm{N} 1$ s core levels consistently suggest different mechanisms of oxygen vacancy formation in PVD- and ALD-grown $\mathrm{HfO}_{2}$ thin films and, in the case of ALD, their electronic interaction with nitrogen impurities, which may directly correlate with the $\mathrm{HfO}_{2}$ ferroelectric properties. By linking the HAXPES results to electric field cycling experiments on the TiN/ $\mathrm{HfO}_{2} / \mathrm{TiN}$ capacitors, a novel view on how the stabilization of the ferroelectric $\mathrm{HfO}_{2}$ phase directly relates to specific PVD or ALD growth conditions is provided. Hence, our proposed model may inspire further studies on the complex electronic interactions between oxygen vacancies $\left(\mathrm{V}_{0}\right)$ and impurities $(\mathrm{C}$ or $\mathrm{N})$ in ferroelectric $\mathrm{HfO}_{2}$ and their impact on electric characteristics of $\mathrm{TiN} / \mathrm{HfO}_{2} / \mathrm{TiN}$ capacitors.

See the supplementary material for details about materials growth and crystallization as well as additional ferroelectric and HAXPES results.

This project received funding from the European Union's Horizon 2020 research and innovation programme under Grant Agreement No. 780302. We acknowledge DESY (Hamburg, Germany), a member of the Helmholtz Association HGF, for the provision of experimental facilities. Funding for the HAXPES instrument at beamline P22 by the Federal Ministry of Education and Research (BMBF) under Contract Nos. 05KS7UM1 and 05K10UMA with Universität Mainz; Nos. 05KS7WW3, $05 \mathrm{~K} 10 \mathrm{WW} 1$, and $05 \mathrm{~K} 13 \mathrm{WW} 1$ with Universität Würzburg is gratefully acknowledged.

\section{DATA AVAILABILITY}

The data that support the findings of this study are available from the corresponding author upon reasonable request.

\section{REFERENCES}

${ }^{1}$ T. S. Böscke, J. Müller, D. Bräuhaus, U. Schröder, and U. Böttger, "Ferroelectricity in hafnium oxide thin films," Appl. Phys. Lett. 99, 102903 (2011).

${ }^{2}$ A. Rodriguez-Fernandez, C. Cagli, L. Perniola, E. Miranda, and J. Suñé, "Characterization of $\mathrm{HfO}_{2}$-based devices with indication of second order memristor effects," Microelectron. Eng. 195, 101-106 (2018).

${ }^{3}$ E. Covi, S. Brivio, A. Serb, T. Prodromakis, M. Fanciulli, and S. Spiga, " $\mathrm{HfO}_{2}$ based memristors for neuromorphic applications," in IEEE International Symposium on Circuits and Systems (ISCAS) (2016), pp. 393-396.

${ }^{4}$ T. Mittmann, M. Materano, P. D. Lomenzo, M. H. Park, I. Stolichnov, M. Cavalieri, C. Zhou, C.-C. Chung, J. L. Jones, T. Szyjka, M. Müller, A. Kersch, T. Mikolajick, and U. Schroeder, "Origin of ferroelectric phase in undoped $\mathrm{HfO}_{2}$ films deposited by sputtering,” Adv. Mater. Interfaces 6, 1901528 (2019).

${ }^{5}$ M. Pešić, F. P. G. Fengler, L. Larcher, A. Padovani, T. Schenk, E. D. Grimley, X. Sang, J. M. LeBeau, S. Slesazeck, U. Schroeder, and T. Mikolajick, "Physical mechanisms behind the field-cycling behavior of $\mathrm{HfO}_{2}$-based ferroelectric capacitors," Adv. Funct. Mater. 26, 4601-4612 (2016).

${ }^{6} \mathrm{R}$. Materlik, C. Künneth, and A. Kersch, "The origin of ferroelectricity in $\mathrm{Hf}_{1-x} \mathrm{Zr}_{\mathrm{x}} \mathrm{O}_{2}$ : A computational investigation and a surface energy model," J. Appl. Phys. 117, 134109 (2015).

${ }^{7}$ M. Müller, S. Nemšák, L. Plucinski, and C. M. Schneider, "Functional materials for information and energy technology: Insights by photoelectron spectroscopy," J. Electron Spectrosc. Related Phenom. 208, 24-32 (2016). 
${ }^{8}$ T. Gerber, P. Lömker, B. Zijlstra, C. Besson, D. N. Mueller, W. Zander, J. Schubert, M. Gorgoi, and M. Müller, "Thermodynamic stability and control of oxygen reactivity at functional oxide interfaces: Euo on ITO," J. Mater. Chem. C 4, 1813-1820 (2016).

${ }^{9}$ T. Szyjka, L. Baumgarten, T. Mittmann, Y. Matveyev, C. Schlueter, T. Mikolajick, U. Schroeder, and M. Müller, "Enhanced ferroelectric polarization in Tin/ $\mathrm{HfO}_{2} /$ Tin capacitors by interface design," ACS Appl. Electron. Mater. 2, 3152-3159 (2020).

${ }^{10}$ W. Hamouda, A. Pancotti, C. Lubin, L. Tortech, C. Richter, T. Mikolajick, U. Schroeder, and N. Barrett, "Physical chemistry of the TiN/Hf ${ }_{0.5} \mathrm{Zr}_{0.5} \mathrm{O}_{2}$ interface," J. Appl. Phys. 127, 064105 (2020).

${ }^{11}$ K. Xiong, J. Robertson, M. C. Gibson, and S. J. Clark, "Defect energy levels in $\mathrm{Hfo}_{2}$ high-dielectric-constant gate oxide,” Appl. Phys. Lett. 87, 183505 (2005).

${ }^{12} \mathrm{P}$. Broqvist and A. Pasquarello, "Oxygen vacancy in monoclinic $\mathrm{HfO}_{2}$ : A consistent interpretation of trap assisted conduction, direct electron injection, and optical absorption experiments," Appl. Phys. Lett. 89, 262904 (2006).

${ }^{13}$ J. L. Gavartin, D. Muñoz Ramo, A. L. Shluger, G. Bersuker, and B. H. Lee, "Negative oxygen vacancies in $\mathrm{HfO}_{2}$ as charge traps in high-k stacks," Appl. Phys. Lett. 89, 082908 (2006).

${ }^{14}$ S.-J. Yoon, S.-Y. Na, S.-E. Moon, and S.-M. Yoon, "Polarization switching kinetics of the ferroelectric Al-doped $\mathrm{HfO}_{2}$ thin films prepared by atomic layer deposition with different ozone doses," J. Vac. Sci. Technol. B 37, 050601 (2019).

${ }^{15}$ D. J. J. Loy, P. A. Dananjaya, S. Chakrabarti, K. H. Tan, S. C. W. Chow, E. H. Toh, and W. S. Lew, "Oxygen vacancy density dependence with a hopping conduction mechanism in multilevel switching behavior of $\mathrm{HfO}_{2}$-based resistive random access memory devices," ACS Appl. Electron. Mater. 2, 3160 (2020).

${ }^{16}$ S. Jain, J. Shah, N. S. Negi, C. Sharma, and R. K. Kotnala, "Significance of interface barrier at electrode of hematite hydroelectric cell for generating ecopower by water splitting," Int. J. Energy Res. 43, 4743-4755 (2019).

${ }^{17}$ J.-C. Dupin, D. Gonbeau, P. Vinatier, and A. Levasseur, "Systematic XPS studies of metal oxides, hydroxides and peroxides," Phys. Chem. Chem. Phys. 2, 1319-1324 (2000).

${ }^{18}$ R. Zhang, H. Huang, Q. Xia, C. Ye, X. Wei, J. Wang, L. Zhang, and L. Q. Zhu, "Role of oxygen vacancies at the $\mathrm{TiO}_{2} / \mathrm{HfO}_{2}$ interface in flexible oxide-based resistive switching memory," Adv. Electron. Mater. 5, 1800833 (2019).

${ }^{19}$ Y. Lai, Z. Zeng, C. Liao, S. Cheng, J. Yu, Q. Zheng, and P. Lin, "Ultralow switching current in hfox/zno bilayer with tunable switching power enabled by plasma treatment," Appl. Phys. Lett. 109, 063501 (2016).

${ }^{20}$ C.-Y. Huang, C.-Y. Huang, T.-L. Tsai, C.-A. Lin, and T.-Y. Tseng, "Switching mechanism of double forming process phenomenon in $\mathrm{ZrO}_{\mathrm{x}} / \mathrm{HfO}_{\mathrm{y}}$ bilayer resistive switching memory structure with large endurance," Appl. Phys. Lett. 104, 062901 (2014).

${ }^{21}$ F. P. G. Fengler, R. Nigon, P. Muralt, E. D. Grimley, X. Sang, V. Sessi, R. Hentschel, J. M. LeBeau, T. Mikolajick, and U. Schroeder, "Analysis of performance instabilities of hafnia-based ferroelectrics using modulus spectroscopy and thermally stimulated depolarization currents," Adv. Electron. Mater. 4, 1700547 (2018).
${ }^{22}$ S. Karwal, M. A. Verheijen, B. L. Williams, T. Faraz, W. M. M. Kessels, and M. Creatore, "Low resistivity $\mathrm{HfN}_{\mathrm{x}}$ grown by plasma-assisted ALD with external rf substrate biasing," J. Mater. Chem. C 6, 3917-3926 (2018).

${ }^{23}$ C. Zheng, G. He, X. Chen, M. Liu, J. Lv, J. Gao, J. Zhang, D. Xiao, P. Jin, S. Jiang, W. Li, and Z. Sun, "Modification of band alignments and optimization of electrical properties of InGaZnO MOS capacitors with high-k $\mathrm{HfO}_{\mathrm{x}} \mathrm{N}_{\mathrm{y}}$ gate dielectrics," J. Alloys Compd. 679, 115-121 (2016).

${ }^{24}$ K. D. Kim, M. H. Park, H. J. Kim, Y. J. Kim, T. Moon, Y. H. Lee, S. D. Hyun, T. Gwon, and C. S. Hwang, "Ferroelectricity in undoped- $\mathrm{HfO}_{2}$ thin films induced by deposition temperature control during atomic layer deposition," J. Mater. Chem. C 4, 6864-6872 (2016)

${ }^{25}$ C. Rodenbücher, E. Hildebrandt, K. Szot, S. U. Sharath, J. Kurian, P. Komissinskiy, U. Breuer, R. Waser, and L. Alff, "Hafnium carbide formation in oxygen deficient hafnium oxide thin films,” Appl. Phys. Lett. 108, 252903 (2016).

${ }^{26}$ L. Xu, T. Nishimura, S. Shibayama, T. Yajima, S. Migita, and A. Toriumi, "Ferroelectric phase stabilization of $\mathrm{HfO}_{2}$ by nitrogen doping," Appl. Phys. Express 9, 091501 (2016).

${ }^{27}$ Y. Zhou, Y. Zhang, Q. Yang, J. Jiang, P. Fan, M. Liao, and Y. Zhou, "The effects of oxygen vacancies on ferroelectric phase transition of $\mathrm{HfO}_{2}$-based thin film from first-principle," Comput. Mater. Sci. 167, 143- 150 (2019).

${ }^{28}$ N. Umezawa, K. Shiraishi, T. Ohno, H. Watanabe, T. Chikyow, K. Torii, K. Yamabe, K. Yamada, H. Kitajima, and T. Arikado, "First-principles studies of the intrinsic effect of nitrogen atoms on reduction in gate leakage current through Hf-based high-k dielectrics," Appl. Phys. Lett. 86, 143507 (2005).

${ }^{29}$ N. Umezawa, K. Shiraishi, Y. Akasaka, A. Oshiyama, S. Inumiya, S. Miyazaki, K. Ohmori, T. Chikyow, T. Ohno, K. Yamabe, Y. Nara, and K. Yamada, "Chemical controllability of charge states of nitrogen-related defects in $\mathrm{HfO}_{\mathrm{x}} \mathrm{N}_{\mathrm{y}}$ : First-principles calculations,” Phys. Rev. B 77, 165130 (2008).

${ }^{30} \mathrm{~K}$. Xiong, J. Robertson, and S. J. Clark, "Passivation of oxygen vacancy states in $\mathrm{HfO}_{2}$ by nitrogen,” J. Appl. Phys. 99, 044105 (2006).

${ }^{31}$ C. Lee, J. Choi, M. Cho, J. Park, C. S. Hwang, H. J. Kim, and J. Jeong, "Nitrogen incorporation engineering and electrical properties of high-k gate dielectric $\left(\mathrm{HfO}_{2}\right.$ and $\left.\mathrm{Al}_{2} \mathrm{O}_{3}\right)$ films on $\mathrm{Si}(100)$ substrate," J. Vac. Sci. Technol., B 22, $1838-1843$ (2004).

${ }^{32}$ J. H. Kim, T. J. Park, M. Cho, J. H. Jang, M. Seo, K. D. Na, C. S. Hwang, and J. Y. Won, "Reduced electrical defects and improved reliability of atomic-layerdeposited $\mathrm{HfO}_{2}$ dielectric films by in situ $\mathrm{NH}_{3}$ injection," J. Electrochem. Soc. 156, G48 (2009).

${ }^{33}$ P. D. Lomenzo, Q. Takmeel, C. Zhou, C. M. Fancher, E. Lambers, N. G. Rudawski, J. L. Jones, S. Moghaddam, and T. Nishida, "Tan interface properties and electric field cycling effects on ferroelectric Si-doped $\mathrm{HfO}_{2}$ thin films," T. Appl. Phys. 117, 134105 (2015).

${ }^{34}$ M. Hoffmann, U. Schroeder, T. Schenk, T. Shimizu, H. Funakubo, O. Sakata, D. Pohl, M. Drescher, C. Adelmann, R. Materlik, A. Kersch, and T. Mikolajick, "Stabilizing the ferroelectric phase in doped hafnium oxide," J. Appl, Phys. 118, 072006 (2015).

${ }^{35}$ S. Starschich, S. Menzel, and U. Böttger, "Evidence for oxygen vacancies movement during wake-up in ferroelectric hafnium oxide," Appl. Phys. Lett. 108, 032903 (2016). 